\section{International Conference on Carbon Nanotubes continues to explore new research directions}

nt13.aalto.fi

$\mathbf{T}$ he Fourteenth International Conference on Carbon Nanotubes (NT13) took place at Aalto University in Espoo, Finland, on June 24-28, 2013. The conference - chaired by Esko Kauppinen, Risto Nieminen, and Pertti Hakonen of Aalto-revealed continuous, significant progress in this research area, with 440 attendees representing 34 countries. Toma Susi of Aalto headed the local organizing committee.

By remaining focused on nanotubes and allowing graphene to enrich nanotube research, this conference series has maintained its unique offerings to the field. Using graphene as a substrate for nanotubes adds new functionality to carbon nanotubes and should be pursued. From a spectroscopic standpoint the enhancement effects have made it possible to observe and study in detail features that were not readily observable previously. Based on the presentations, connections between the different-layered graphenes (1LG, 2LG, 3LG) and multiwalled nanotubes (SWNTs, DWNTs, TWNTs) seem like a fruitful research direction for the future. One area that has grown is applications, for example, the use of carbon nanotubes for water purification applications with potential societal impact in developing countries. The use of nanotube-graphene-polymer composites ranges from advanced materials applications for indium-tin-oxide (ITO) replacement to various other sensitive probe devices, and start-up company development based on this type of composite materials research is expected to increase significantly in the coming years.

Nanotube growth remains a dominant challenge for all nanoscience, since property measurements are often limited by the quality of available materials. The cloning approach to nanotube growth for increased diameter and chirality control, advocated many years ago by Richard
Smalley of Rice University, is now gaining increased interest by the research community, with the recent demonstration of the feasibility of this approach in the laboratory. At NT13 we heard reports on the systematic study of $(7,6)$, $(6,5)$, and $(7,7)$ tubes using the cloning method at the University of Southern California, and device prototypes that were made using the cloning principles. The study of the difference in growth rate for various types of tubes is an area for future work to support applications developments in industry. It is quite early to determine which new approaches will make the cloning approach practical.

The complementary use of theory and experiment to increase understanding and to suggest new research directions has long been successfully practiced in nanotube research. The reports on such successful collaborations in interpreting nanotube filling experiments involving two strands were particularly impressive at NT13. Future nanotube research opportunities might come from the use of results from folded bilayer graphene
Fullerene, graphene, and nanotube publications. at special folding angles where moiré patterns occur. Further understanding of double- and triple-walled carbon nanotube stacking may be thus gained where curvature effects in nanotubes add complexity to twisting interlayer effects that are more simply observed in graphene. Now that serious investigation has started with studying the nanowire structures formed within the hollow core of carbon nanotubes, serious consideration should be given to suggesting new and interesting nanostructural filling materials into the cavities to build on the excellent work that has been accomplished thus far.

Whereas filling nanotubes with water was previously highlighted in this conference series, filling tubes with many different chemical species was reported at the 2013 conference. In particular, Eu was reported to be most effective in forming atomically thin metallic nanowires. We heard that any species that would easily enter a nanotube could be used to form an internal nanowire in the nanotube central channel. Here the nanotube channel acts as an effective internal chemical chamber providing stabilized nanowire growth in an impressive way, as calculated by David Tomanek of Michigan State University and demonstrated experimentally by Hisanori Shinohara of Nagoya University. Not only was a tube filled with Eu seen to form a nanowire, the filling was at

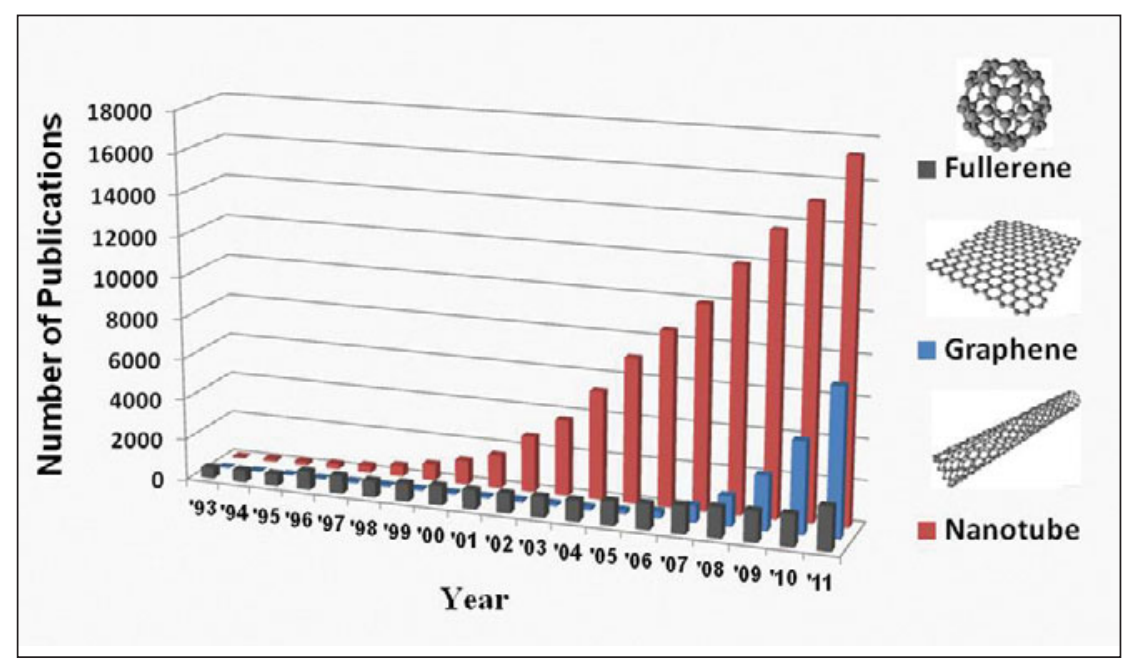


$80-90 \%$ by selected nanowires on average. Theory also suggested filling the inner core of a nanotube with a diamond nanowire, which was successfully demonstrated by Shinohara using a diamondoid precursor. More theory is now needed to account for the detailed results that were actually obtained, such as the formation of two thin Eu nanowires rather than a single large-diameter nanowire, and to explain why these nanowires wrap around each other, rather than forming a straight aligned pair. Theory should also provide an incentive for attempting other new filling configurations with new interesting structures and filling materials. Furthermore, what systematic studies of rare-earth nanowires can be made that would bring new understanding either of electron transport or maybe also spin transport in such nanowires? Maybe temperature dependence or pressure dependence could cause different crystallographic structures, giving rise to new research directions.

Applications of carbon nanotubes to other scientific disciplines played a much greater role at NT13 than before. In biological applications, we heard about the use of nanotubes to penetrate the walls of cells, with biological cargo delivered to the cells through the nanotube channels, a promising growing interdisciplinary research field for biophysics. The study of specific enzymes at the single-enzyme level studied from a nanotube sensor viewpoint was also featured, and suggests development of interdisciplinary group activities between biologists, chemists, physicists, and materials scientists, as promoted by the US National Institutes of Health. The study of biological damage for medical applications is another research direction that is occurring. The injection of singlewalled carbon nanotubes into lung and neuron cells has been found to be damaging for SWNT lengths in excess of 200 $\mathrm{nm}$. Innovations like ionic liquids when combined with carbon nanotubes have been shown to provide novel functionality when used with electrolytes, and for increasing the flexibility and stretchability of materials used in supercapacitors. Nanocarbons have for some time been used for battery applications, but at NT13 we saw new uses of nanotubes to increase the performance of battery materials, as well as of carbon fiber yarn materials. Other examples of the use of nanotubes in other fields of science include their use as active probes in plas- monics, in increasing the sensitivity in piezoresistance measurements, in scaleup of piezoresistance technology applications, and to microelectromechanical systems technology at the nanoscale.

These are just a few of the highlights presented at NT13. In addition, satellite workshops were held on more focused topics, such as nanometrology as reported by Ado Jorio in this issue of MRS Bulletin. The interaction between theoretical and experimental research to advance research frontiers should be further promoted to accelerate the progress of nanotube research into new directions. Increasing interest in carbon nanotubes globally follows general international growth trends in nanotube research (see Figure). Remarkably, as the graph shows, while graphene came onto the scene with such fervor, advances in nanotube research also continue to flourish. Due to Europe's Graphene Flagship program that started in the Fall of 2013, we may expect in the future to more regularly see novel examples of graphene/ nanotube hybrid structures presented at international conferences.

Mildred S. Dresselhaus,

Massachusetts Institute of Technology, USA

\section{Nanometrology satellite workshop reveals significant progress} nt13.aalto.fi/satellites/MSIN13

A bout six years ago, the international ISO Standards Committee began emphasizing carbon nanotubes and related materials as a prototype material for establishing nanometrology standards. These standards are needed in order to transition the achievements in nanoscience to nanotechnology. Due to the different properties exhibited by materials in bulk form as compared to the nanoscale, the research community recognizes the need for establishing metrology on the nanoscale level. In view of this need, the International Conference on Carbon Nanotubes established a satellite series of workshops on this topic. The Sixth International Workshop on Metrology, Standardization and Industri- al Quality of Nanotubes (MSIN13) took place in Tallinn, Estonia, on June 29.

For synthesis and processing, the advances on separation of single-walled carbon nanotubes by diameter, metallicity, and chiral angle show that chemistry has a key role in adding value to nanotechnology. It appears that separation is no longer the cost limit of carbon nanotube products. Further reduction in manufacturing costs for bulk production is needed. In this context, exfoliation of graphene from natural graphite may have an advantage.

Separation procedures by diameter and chirality are likely to play an important role in the development of standard reference materials for characterizing nanotubes. Interestingly, the application of carbon-related standards can be used for developing nanometrology in general, with graphene being proposed as a reference material for characterizing the limiting properties of focused ionbeam devices, which were widely used for nanopatterning.

On the issue of quality and evaluation protocols, characterization protocols proposed in early reports of MSIN meetings are already being considered as standard techniques, as they are being broadly used for purity and quality evaluation. Commonly used characterization techniques are electron microscopy, thermo gravimetric analysis, Raman spectroscopy, and optical absorp- 